\title{
TITLE:
}

\section{Ergodic Partition of Phase Space in Continuous Dynamical Systems}

$\operatorname{AUTHOR}(\mathrm{S})$ :

Susuki, Yoshihiko; Mezi, Igor

\section{CITATION:}

Susuki, Yoshihiko ... [et al]. Ergodic Partition of Phase Space in Continuous Dynamical Systems. Proceedings of the Joint 48th IEEE Conference on Decision and Control and 28th Chienese Control Conference (CDC/CCC2009) 2009: 7497-7502

\section{ISSUE DATE:}

2009-12

URL:

http://hdl.handle.net/2433/89656

\section{RIGHT:}

(c) 2009 IEEE. Personal use of this material is permitted. However, permission to reprint/republish this material for advertising or promotional purposes or for creating new collective works for resale or redistribution to servers or lists, or to reuse any copyrighted component of this work in other works must be obtained from the IEEE. 


\title{
Ergodic Partition of Phase Space in Continuous Dynamical Systems
}

\author{
Yoshihiko Susuki and Igor Mezić
}

\begin{abstract}
The theory of ergodic partition of phase space in discrete dynamical systems is extended to continuous dynamical systems or flows. This makes it possible to identify invariant sets of measure-preserving flows such as Hamiltonian flows. The extended theory is applied to an analysis of transient stability of multi-machine power systems.
\end{abstract}

\section{INTRODUCTION}

Ergodicity is a well-established subject with a long history of research in dynamical systems [1], [2]. The notion of ergodicity is that there are no invariant sets of positive measure with measure less than that of (a compact manifold of) phase space [3]. It originates from foundation of statistical physics and, apart from the physical origin, has been extensively studied in the theory of dynamical systems. The so-called ergodic theory of dynamical systems is applied to control engineering, for example, control of mixing of fluid flows [4] and nonlinear control [5], [6].

A method for visualization of invariant sets of discrete dynamical systems possessing a smooth invariant measure is developed in [7]. This is based on the so-called ergodic partition or ergodic decomposition [8]. This method partitions a compact metric phase space $M$ with the level sets of time averages of a set of functions defined on $M$ and identify invariant sets in $M$ on which dynamics are ergodic. In an ergodic invariant set, all points are accessible in the sense that the initial conditions in this set thoroughly sample the set. The method provides an effective tool for analyzing complex dynamics described by discrete-time nonlinear models. The theory is being developed in [9], [10], [11].

This paper is concerned with the existing theory of ergodic partition and its application to an engineering problem. The purpose of this paper is twofold.

One is to extend the theory of ergodic partition developed in [7] to continuous dynamical systems or flows. The extended theory is applicable to analysis of dynamics described by continuous-time nonlinear models. Examples of the application include dynamics of oscillators excited by multiple frequencies [12], which case is studied in this paper, and fluid dynamics with time-dependent velocity profiles. In the farmer part of this paper (Sec. III), we develop the theory

This research is supported in part by the JSPS Postdoctoral Fellowships for Research Abroad.

Y. Susuki is with the Department of Mechanical Engineering at the University of California, Santa Barbara, CA 93106-5070 USA, and with the Department of Electrical Engineering at Kyoto University, Katsura, Nishikyo, Kyoto 615-8510 Japan (e-mail: susuki@ieee.org).

I. Mezić is with the Department of Mechanical Engineering at the University of California, Santa Barbara, CA 93106-5070 USA (e-mail: meziclengineering.ucsb.edu). of ergodic partition for measure-preserving flows that is a natural extension of [7].

The other is to apply the obtained theory to an analysis of transient stability of multi-machine power systems. In [13], [14], we uncovered a novel instability of short-term (0 to 10 seconds) swing dynamics that we termed the Coherent Swing Instability (CSI). CSI is an undesirable and emergent phenomenon of synchronous machines in a power system, in which most of the machines in the system coherently lose synchronism with the rest of the system after being subjected to a finite disturbance. This phenomenon does not happen upon an infinitesimally small perturbation around an equilibrium of nonlinear swing equations. However, it encompasses the situation when the system escapes a predefined set around the equilibrium. In this way, the notion of instability that we address here is non-local. In [13], [14], we derived a reduced-order, planar dynamical system that described averaged dynamics of machines in a simple loop power system and characterized the non-local instability. The planar system has quasi-periodically external excitations (so it is non-autonomous), and its solutions define a measurepreserving flow. In the later part of this paper (Sec. IV), by applying the theory developed in Sec. III to this flow, we numerically identify invariant sets on two-dimensional plane of initial conditions at one initial time. These sets are bounded on the initial conditions' plane and represent safe operating conditions of the loop power system in which all synchronous machines show bounded swings. Furthermore, we theoretically show that the invariant sets are uniformly bounded in the sense that the boundedness property does not depend on initial time. This implies that CSI for the loop power system occurs when an initial condition of the planar system is placed outside a family of uniformly-bounded invariant sets. Thus we suggest that such an invariant structure could be related to the mechanism of CSI occurring for multi-machine power systems.

\section{Measure-Preserving Flow and Partitions of Phase SPACE}

We consider a measure-preserving flow of a probability space. A probability space is the tuple $\left(M, \mathcal{B}_{M}, \mu\right)$ where $X$ is a compact metric space, $\mathcal{B}_{M}$ is the Borel $\sigma$-algebra of $M$, and $\mu$ is a probability measure. A measure-preserving flow of the probability space is a 1-parameter group of measurepreserving diffeomorphisms $\left\{\phi_{t}: t \in \mathbb{R}\right\}$ such that each $\phi_{t}$ is measure-preserving ${ }^{1}$, and $\phi_{0}: M \rightarrow M$ the identity and $\phi_{t+s}=\phi_{t} \circ \phi_{s}$.

${ }^{1} \mu\left(\phi_{-t}(B)\right)=\mu(B)$ for all $B \in \mathcal{B}_{M}$ 
The important notion that we address here is the partition of phase space. The definitions of partition and measurable partition are as follows (we denote by $B^{\mathrm{c}}$ the complementary set to a subset $B$ of $M)$ :

Definition 1 (Partition and Measurable Partition [7]): A family $\zeta$ of disjoint sets whose union is identically $M$ is called a partition of $M$. Let $A_{\alpha}$ be an element of $\zeta$ where $\alpha$ is an element of some indexing set $A$. The partition $\zeta$ is called measurable if there exists a countable family $\Delta$ of measurable sets $\left\{B_{i}\right\}$ such that (i) every $B_{i}$ is a union of elements of $\zeta$, and (ii) for every pair $A_{\alpha}, A_{\beta}$ of elements of $\zeta$, there exists $B \in \Delta$ such that $A_{\alpha} \subset B$ and $A_{\beta} \subset B^{\mathrm{c}}$.

In Sec. III we use a product operation on many different measurable partitions of $M$. The product operation is defined as follows:

Definition 2 (Product of Partitions [7]): Let $\zeta_{1}$ and $\zeta_{2}$ be two measurable partitions of $M$, let $A_{1}$ and $A_{2}$ be elements of $\zeta_{1}$ and $\zeta_{2}$, respectively, and let $\zeta$ be the family of all sets of the form $A=A_{1} \cap A_{2}$. The partition $\zeta$ is also measurable and is called a product of partitions $\zeta_{1}$ and $\zeta_{2}$, denoted by $\zeta=\zeta_{1} \vee \zeta_{2}$.

For a finite or countable product, we denote it by $\zeta=\bigvee_{i=1}^{n} \zeta_{i}$ for finite $n$ or $\infty$.

\section{Ergodic Partition under Measure-Preserving FLOW}

We extend the theory of ergodic partition in [7] to measure-preserving flows. This section consists of a few lemma and theorem. Their proofs almost coincide with the existing proofs in [7] and are omitted in this paper. In what follows, we denote by $L^{1}(M)$ the space of all realvalued, $\mu$-integrable functions on $M$, by $C(M)$ the set of all real-valued, continuous functions on $M$, and by $S$ a dense countable subset of $C(M)$.

The so-called ergodic partition is the heart of our developing theory: roughly speaking, partition of the phase space $M$ into (invariant) sets on which $\phi_{t}$ is ergodic. The precise definition is as follows:

Definition 3 (Ergodic Partition): For a measurable partition $\zeta$ of $M$, let $A_{\alpha}$ be an element of $\zeta$ where $\alpha$ is an element of some indexing set $A$ as a measure space $\left(A, \mathcal{B}_{A}, P\right)$. The partition $\zeta$ is called ergodic under $\phi$ if (i) for almost every (with respect to $\mu$ ) element $A_{\alpha}$, it is invariant for $\phi$ and there exists an invariant probability measure $\mu_{A_{\alpha}}$ on it such that for every $f \in L^{1}(M)$,

$$
\lim _{T \rightarrow \infty} \frac{1}{T} \int_{0}^{T}\left(f \circ \phi_{t}\right)(x) \mathrm{d} t=\int_{A_{\alpha}} f \mathrm{~d} \mu_{A_{\alpha}},
$$

for almost everywhere (a.e.) with respect to $\mu_{A_{\alpha}}$ on $A_{\alpha}$, and (ii) for every $f \in L^{1}(M)$,

$$
\int_{M} f \mathrm{~d} \mu=\int_{A}\left[\int_{A_{\alpha}} f \mathrm{~d} \mu_{A_{\alpha}}\right] \mathrm{d} P(\alpha) .
$$

Here we call the function $f^{*}$ the time average of a function $f$ under $\phi$ if

$$
f^{*}(x)=\lim _{T \rightarrow \infty} \frac{1}{T} \int_{0}^{T}\left(f \circ \phi_{t}\right)(x) \mathrm{d} t,
$$

a.e. on $M$. Birkhoff's pointwise ergodic theorem [15], [1] shows that for every $f \in L^{1}(M)$, (i) $f^{*}$ exists; (ii) $f^{*} \circ \phi_{\tau}=$ $f^{*}$ for a.e. on $M$ and every $\tau \in \mathbb{R}$; and (iii) $\int_{M} f \mathrm{~d} \mu=$ $\int_{M} f^{*} \mathrm{~d} \mu$. We denote by $\Sigma$ the set of all $x \in M$ such that $f^{*}(x)$ exists for every $f \in C(M)$, and by $\Sigma(f)$ the set of all $x \in M$ such that $f^{*}(x)$ exists for a particular $f \in C(M)$. The following lemma is standard.

Lemma 1 (Mezić and Wiggins [7]):

$$
\Sigma=\bigcap_{f \in S} \Sigma(f) .
$$

Now consider the set $\Sigma$ on which the time averages of all continuous functions on $M$ are well-defined on $\Sigma$. The complimentary set $\Sigma^{\mathrm{c}}$ is of measure zero. This is because by the ergodic theorem (i) each $\Sigma(f)^{\mathrm{c}}$ is of measure zero, and thus $\Sigma^{\mathrm{c}}:=\bigcup_{f \in S} \Sigma(f)^{\mathrm{c}}$ is the countable union of measure zero sets, which is again of measure zero. The next lemma shows that the time average of a measurable and bounded function induces a measurable partition on $\Sigma$.

Lemma 2: Let $f$ be a continuous function on $M$. The family of sets $\left\{A_{\alpha}\right\}_{\alpha \in \mathbb{R}}$, where $A_{\alpha}:=\left(f^{*}\right)^{-1}(\alpha)$, is a measurable partition of $\Sigma$.

We denote this partition by $\zeta_{f}$ and call it the partition induced by $f$.

We are in a position to state the first main theorem in this paper. The theorem says that $\zeta_{f}$ induces an ergodic partition of $M$.

Theorem 1: Let $\zeta_{\mathrm{e}}$ be the product of measurable partitions of $\Sigma$ induced by every $f \in S$,

$$
\zeta_{\mathrm{e}}=\bigvee_{f \in S} \zeta_{f} \text {. }
$$

The partition $\zeta_{\mathrm{e}}$ together with $\Sigma^{\mathrm{c}}$ is ergodic under $\phi$.

Now the sets $A_{\alpha}=\left\{x \in X: x \in \Sigma, f^{*}(x)=\alpha\right\}$ for every $\alpha \in \mathbb{R}$ are invariant under $\phi$ on which the dynamics are ergodic. Mezić and Wiggins [7] used the level sets $A_{\alpha}$ based on the time average of $f$ to identify invariant sets in discrete dynamical systems. In this paper we apply the developed theory of ergodic partition to investigate an instability phenomenon occurring for multi-machine power systems.

\section{Application to Power System Analysis}

We apply the theory of ergodic partition in Sec. III to an analysis of short-term swing dynamics, which we term the Coherent Swing Instability (CSI), in a multi-machine power system.

\section{A. The Mathematical Model}

Consider short-term swing dynamics in a simple lossless power system with strong loop transmission network in Fig. 1. The blue circle stands for synchronous generator. 


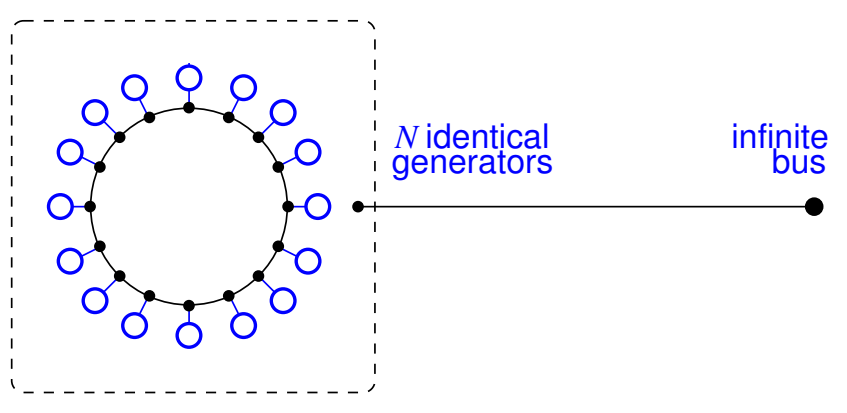

Fig. 1. Simple loop power system [13]

The loop power system consists of $N$ small, identical generators, encompassed by the dotted box, which operate in the loop network and are connected to the infinite bus ${ }^{2}$. The transmission lines joining the infinite bus and a generator are much longer than those joining two generators in the loop network. The lengths of transmission lines between generators are identical. In the preceding papers [13], [14], we demonstrated the CSI phenomenon for the loop power system and developed the following planar dynamical system that characterized the phenomenon:

$$
\left.\begin{array}{rl}
\frac{\mathrm{d} \delta}{\mathrm{d} t} & =\omega, \\
\frac{\mathrm{d} \omega}{\mathrm{d} t} & =p_{\mathrm{m}}-\frac{b}{N} \sum_{i=1}^{N} \sin \left(\sum_{j \in \mathcal{J}} e_{i j} c_{j} \cos \Omega_{j} t+\delta\right),
\end{array}\right\}
$$

where

$$
\begin{aligned}
e_{i j} & =\sqrt{\frac{2}{N}} \cos \left(\frac{2 \pi i j}{N}+\frac{\pi}{4}\right), \\
\Omega_{j} & =2 \sqrt{\left|b_{\text {int }}\right|}\left|\sin \frac{\pi j}{N}\right| .
\end{aligned}
$$

The planar system (2) represents averaged dynamics of the $N$ generators in the loop network. The variable $\delta \in \mathbb{T}^{1}$ is the average of angular positions of rotors with respect to the infinite bus of the $N$ generators, and $\omega \in \mathbb{R}$ the average of deviations of rotor speed in $N$ generators relative to the system angular frequency. The parameter $p_{\mathrm{m}}$ is the mechanical input power to a generator, $b$ the maximum transmission power between the infinite bus and a generator, and $b_{\text {int }}$ the maximum transmission power between two generators in the loop network. The constant $e_{i j}$ is the eigenfunction of mode oscillations occurring in the loop network, $\Omega_{j}$ its eigenfrequency, and $c_{j}$ the strength of mode oscillations. The finite index set $\mathcal{J}$ determines which modes are excited in the loop network.

Solutions of (2) define a measure-preserving flow on phase space $\mathbb{T}^{1} \times \mathbb{R}$. The system (2) is formulated as the following Hamilton's canonical equations:

$$
\frac{\mathrm{d} \delta}{\mathrm{d} t}=\frac{\partial}{\partial \omega} H(\delta, \omega, t), \quad \frac{\mathrm{d} \omega}{\mathrm{d} t}=-\frac{\partial}{\partial \delta} H(\delta, \omega, t),
$$

\footnotetext{
${ }^{2} \mathrm{~A}$ voltage source of constant voltage and constant frequency
}

with the time-dependent Hamiltonian $H(\delta, \omega, t)$, given by

$$
\begin{aligned}
H:= & \frac{1}{2} \omega^{2}-p_{\mathrm{m}} \delta \\
& -\frac{b}{N} \sum_{i=1}^{N} \cos \left(\sum_{j \in \mathcal{J}} e_{i j} c_{j} \cos \Omega_{j} t+\delta\right) .
\end{aligned}
$$

Because the flow defined here is divergence-free, i.e., $(\partial H / \partial \delta)(\mathrm{d} \delta / \mathrm{d} t)+(\partial H / \partial \omega)(\mathrm{d} \omega / \mathrm{d} t)=0$, it is measurepreserving with respect to the Liouville measure $\mathrm{d} \delta \mathrm{d} \omega$. Note that it does not conserve the Hamiltonian $H$, that is, $\mathrm{d} H / \mathrm{d} t \neq 0$ if $c_{j} \neq 0$.

\section{B. Identification of Invariant Sets}

This section applies the technique of ergodic partition to identification of invariant sets in which all the generators show bounded swings. We show in [13], [14] that the divergence motion in the planar system (2) corresponds to the CSI phenomenon. Thus it is important to examine bounded motions of the planar system to understand a dynamical origin of CSI.

Numerical simulations are performed for identification of invariant sets using the ergodic partition. To do so, it needs to fix (i) the set of (infinitely) countable functions, $\left\{f_{n}\right\}_{n \in \mathbb{N}}$, (ii) the subset of phase space on which we find invariant sets, and (iii) the exit time to obtain a good approximation of each time average $f_{n}^{*}$. This paper uses one function $f(\delta)=\sin 2 \delta$ according to the investigation in [7], [9], [10] and the grid of $201 \times 201$ of initial conditions $(\delta, \omega)$ at $t=0$ on $[0, \pi] \times$ $[-0.02,0.02]$ or $[0, \pi] \times[-0.04,0.04]$, and adopts the exit time $t_{\mathrm{ex}}$ below. Numerical integration of the planar system is performed with the 4th explicit symplectic integrator [16] with time step $h$ : see Appendix I for its detail. The parameter settings are as follows:

$$
\begin{gathered}
p_{\mathrm{m}}=0.009, b=0.01, N=20, b_{\mathrm{int}}=1, \\
h=\frac{2 \pi}{\Omega_{1}} \frac{1}{2^{8}}, t_{\mathrm{ex}}=h \times 2^{8} \times 15 .
\end{gathered}
$$

The settings of parameters $\left(p_{\mathrm{m}}, b, N, b_{\mathrm{int}}\right)$ are the same as in [13].

Figure 2 shows numerical results of ergodic partition for the flow. The color bar attached to each figure denotes the value of time average $f^{*}(\delta)$. The colored region of each figure corresponds to the two-dimensional cross section of invariant sets under $t=0$. All trajectories from the same colored region take a common value of $f^{*}(\delta)$. That is, the same colored region corresponds to one invariant set on which the dynamics are ergodic. Especially, all trajectories from the colored region are bounded until the final time $t_{\text {ex }}$. On the other hand, any trajectory from non-colored (white) regions increases unboundedly as time runs. The boundedness of trajectories implies no occurrence of CSI in the loop power system.

Let us summarize several features of Fig. 2. There exists a family of closed curves with common time averages. The family of closed curves, that is, the colored region is bounded 


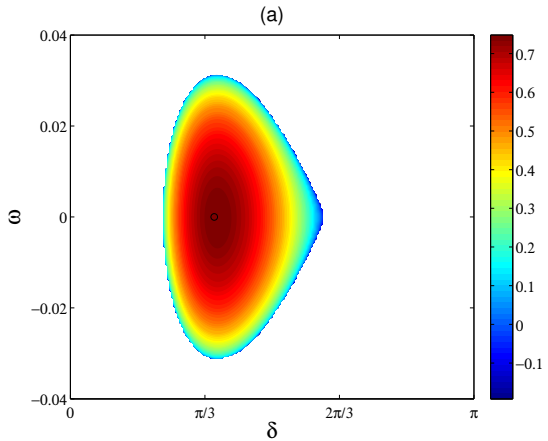

(a) $\mathcal{J}=\{1\}, c_{j}=1$

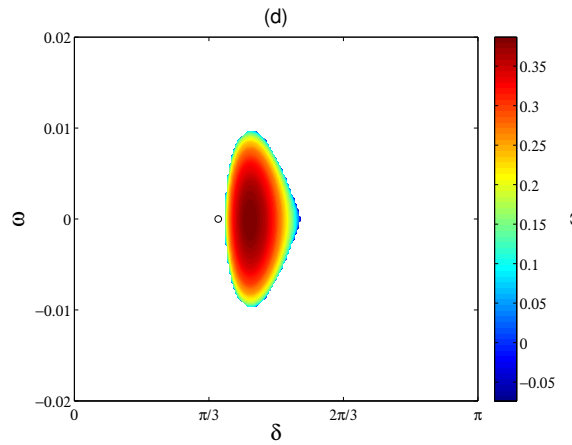

(d) $\mathcal{J}=\{1\}, c_{j}=2.6$

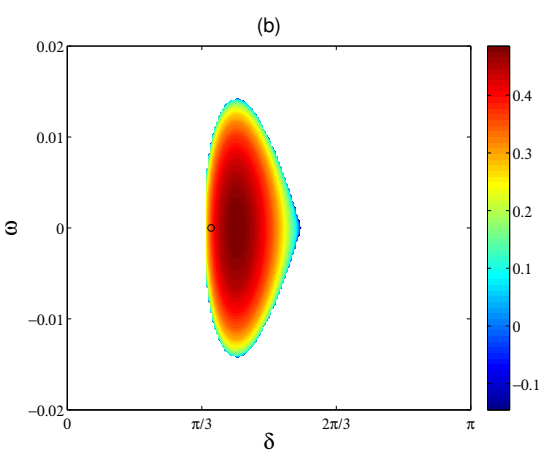

(b) $\mathcal{J}=\{1\}, c_{j}=2.4$

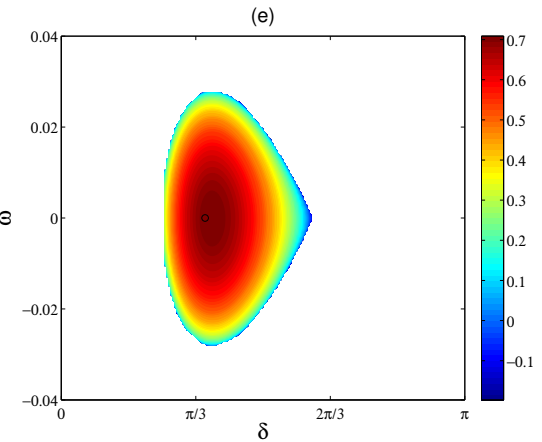

(e) $\mathcal{J}=\{1,2\}, c_{j}=1$

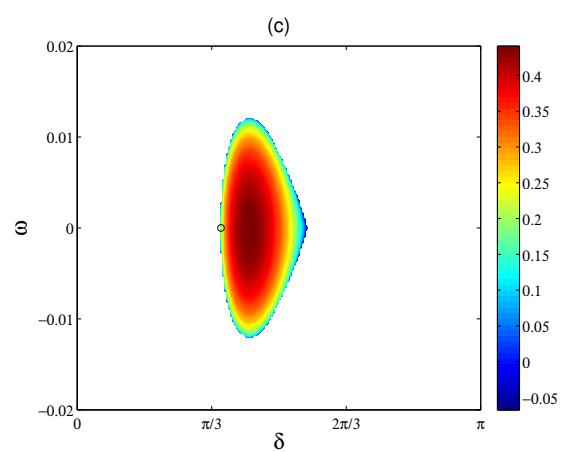

(c) $\mathcal{J}=\{1\}, c_{j}=2.5$

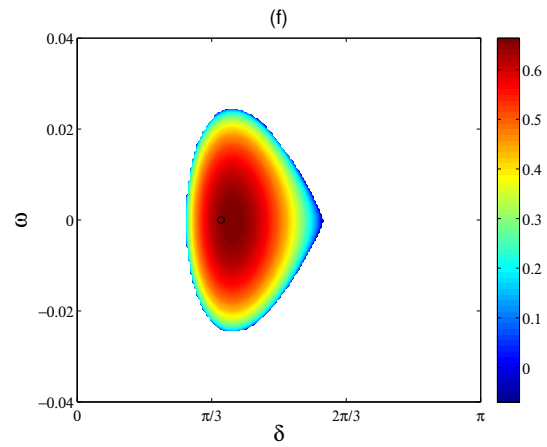

(f) $\mathcal{J}=\{1,2,3\}, c_{j}=1$

Fig. 2. Ergodic partition of phase space in the measure-preserving flow defined by (2). Non-colored (white) regions imply that each trajectory from them diverges as time passes.

in each figure. For the same number of modes in Figs. 2(a)(d), the colored regions shrink as the strength $c_{j}$ increases. This suggests that the strong excitation easily induces the CSI phenomenon for the loop power system. The solid circle (o) on $\omega=0$ represents the condition of normal operation at $c_{j}=0$ for all $j \in \mathcal{J}$, namely, under no external excitation. At $c_{j}=2.5$ in Fig. 2(c) the circle (o) is on the boundary of the colored region, and at $c_{j}=2.6$ in Fig. $2(\mathrm{~d})$ it is outside the colored region. This implies that in Fig. 2(d) we observe the CSI for the initial condition that corresponds to the normal operating condition under no external excitation. This result is consistent with the numerical simulations in [13]. For the same strength $c_{j}$ in Figs. 2(a), (e), and (f), the colored regions also shrink as the number of modes, $k$, increases. This suggests that the excitation based on multiple modes easily induces the CSI phenomenon.

\section{Uniform Boundedness of Invariant Sets}

Section IV-B showed one bounded cross-section of invariant sets under the measure-preserving flow. We confirm that cross-sections of invariant sets are also bounded for other initial time. Thus it is expected that the invariant sets are uniformly bounded in the sense that the boundedness property does not depend on initial time. In this section we prove the uniform boundedness of invariant sets.

Consider the autonomous system obtained by augmenting
(2) in the same way as [12]:

$$
\left.\begin{array}{rl}
\frac{\mathrm{d} \delta}{\mathrm{d} t} & =\omega, \\
\frac{\mathrm{d} \omega}{\mathrm{d} t} & =p_{\mathrm{m}}-\frac{b}{N} \sum_{i=1}^{N} \sin \left(\sum_{j \in \mathcal{J}} e_{i j} c_{j} \cos \theta_{j}+\delta\right), \\
\frac{\mathrm{d} \theta_{j}}{\mathrm{~d} t} & =\Omega_{j},
\end{array}\right\}
$$

where $(\delta, \omega) \in \mathbb{T}^{1} \times \mathbb{R}=: M$ and $\theta_{j} \in \mathbb{T}^{1}$ for each $j \in \mathcal{J}$. The finite cardinality of $\mathcal{J}$ is denoted by $k$. Solutions of (4) define a measure-preserving flow $\phi,\left\{\phi_{t}: t \in\right.$ $\mathbb{R}\}$. We assume that the $k$ excitation frequencies $\left\{\Omega_{j}\right\}_{j \in \mathcal{J}}$ are rationally independent. The augmented phase space $X$ becomes $M \times \mathbb{T}^{k}$. Let $\mathcal{O}\left(x_{0}\right)$ be the orbit through the point $x_{0}$, namely, $\mathcal{O}\left(x_{0}\right):=\left\{x \in X: x=\phi_{t}\left(x_{0}\right), t \in \mathbb{R}\right\}$. We will write $\left.\mathcal{O}\left(x_{0}\right)\right|_{\mathbb{T}^{k}}:=\left\{\theta \in \mathbb{T}^{k}: \theta=\left.\phi_{t}\right|_{\mathbb{T}^{k}}\left(\theta_{0}\right), t \in \mathbb{R}\right\}$, where $x_{0}=\left(m_{0}, \theta_{0}\right) \in M \times \mathbb{T}^{k}$ and $\left.\phi_{t}\right|_{\mathbb{T}^{k}}$ is the restriction of $\phi_{t}$ to $\mathbb{T}^{k}$. Since the $k$ excitation frequencies are rationally independent, we have the following fact:

Fact 1: For any $x \in X,\left.\mathcal{O}(x)\right|_{\mathbb{T}^{k}}$ is dense in $\mathbb{T}^{k}$.

Now we characterize invariant sets in the augmented system (4). Let $I$ be an invariant set under $\phi$. By Fact 1 we have the following decomposition of $I$ :

$$
I=\bigcup_{\theta \in \mathbb{T}^{k}} A_{\theta} \times\{\theta\},
$$

where $A_{\theta}$ is a subset of $M$. Thus we can take a two- 
dimensional cross-section to $I$ for arbitrary $\theta \in \mathbb{T}^{k}$, that is, initial time. Recall that since the numerical results in Sec. IV-B were given for initial conditions $(\delta, \omega)$ at $t=0$, we identified several cross-sections of invariant sets for $\theta_{j}=0$ for all $j \in \mathcal{J}$. Now define a cross-section to $I$ by fixing the variable $\theta$ at $\theta_{0}$ :

$$
I_{\theta_{0}}:=\left\{(m, \theta) \in I: \theta=\theta_{0} \in \mathbb{T}^{k}\right\} .
$$

According to (5), we denote by $I_{\theta_{0}}$ the product set $A_{\theta_{0}} \times$ $\left\{\theta_{0}\right\}$, where $A_{\theta_{0}}$ is a subset of $M$, and by $\mathcal{O}\left(I_{\theta_{0}}\right)$ the set of all orbits from $I_{\theta_{0}},\left\{\mathcal{O}(x): x \in I_{\theta_{0}}\right\}$. Thus we obtain the following theorem that characterizes uniform boundedness of $I$ by means of one sample of it:

Theorem 2: Suppose that $A_{\theta_{0}}$ is bounded in $M$. Then $I$ is bounded in $X$.

Proof: see Appendix II.

Now let us summarize the relationship between uniformlybounded invariant sets and the CSI phenomenon. The instability occurs when the initial condition of (4) (namely (2)) is placed outside a family of invariant sets that are uniformly bounded and represent safe operating conditions of all the machines in the loop power system. The existence of uniformly-bounded invariant sets implies that the CSI can occur in the loop power system.

\section{CONCLUSiOns}

We studied the ergodic partition for continuous dynamical systems. The first contribution is to provide a theory of ergodic partition of phase space in measure-preserving flows that is a natural extension of [7]. The theory is applicable to measure-preserving flows arising in various physical and engineering systems. Examples of the flows include dynamical systems induced by time-dependent Hamiltonians and incompressible fluid flows with time-dependent velocity profiles. The second contribution is to provide a dynamical description of the non-local instability of multi-machine power systems, which we term the Coherent Swing Instability in [13], [14]. The instability occurs when the initial condition of (2) or (4) is placed outside a family of uniformly-bounded invariant sets. Future work is to explore why the uniformlybounded invariant sets change due to the parameter change of quasi-periodic excitation as shown in Fig. 2.

\section{ACKNOWLEDGMENTS}

We are grateful to Professor Takashi Hikihara (Kyoto University) for his encouragement for our research activity and to Mr. Marko Budišić (University of California, Santa Barbara) for his kind introduction to theory and computation of ergodic partition and fruitful discussions.

\section{APPENDIX I}

\section{SyMPLECTIC INTEGRATION OF TIME-DEPENDENT HAMILTONIAN SYSTEMS}

This paper needs to do numerical solutions of the Hamiltonian system (3) with the time-dependent Hamiltonian $H(\delta, \omega, t)$. Symplectic integrator [16] is normally formulated in the case of time-independent Hamiltonians. In this paper we exploit such an integrator by augmenting the original Hamiltonian system. Consider the following Hamiltonian system:

$$
\frac{\mathrm{d} q}{\mathrm{~d} t}=\frac{\partial}{\partial p} H(q, p, t), \quad \frac{\mathrm{d} p}{\mathrm{~d} t}=-\frac{\partial}{\partial q} H(q, p, t),
$$

where $q \in \mathbb{R}^{N}, p \in \mathbb{R}^{N}$, and $t \in \mathbb{R}$. Now, by replacing $t$ with one new variable $q^{\prime}$ and defining the other new variable $p^{\prime}=$ $-\partial H / \partial t$, we have the augmented Hamiltonian $\bar{H}\left(q, p, q^{\prime}, p^{\prime}\right)$ as follows:

$$
\bar{H}\left(q, p, q^{\prime}, p^{\prime}\right):=p^{\prime}+H\left(q, p, q^{\prime}\right) .
$$

Thus we have the augmented Hamiltonian system of the time-independent Hamiltonian system $\bar{H}$ as

$$
\begin{array}{ll}
\frac{\mathrm{d} q}{\mathrm{~d} t}=\frac{\partial \bar{H}}{\partial p}, & \frac{\mathrm{d} p}{\mathrm{~d} t}=-\frac{\partial \bar{H}}{\partial q}, \\
\frac{\mathrm{d} q^{\prime}}{\mathrm{d} t}=\frac{\partial \bar{H}}{\partial p^{\prime}}, & \frac{\mathrm{d} p^{\prime}}{\mathrm{d} t}=-\frac{\partial \bar{H}}{\partial q^{\prime}} .
\end{array}
$$

The flow induced by solutions of this system is divergencefree and conserves the Hamiltonian $\bar{H}$. Thus we can use the integrator for the augmented system and do numerical solutions of the original system. Note that we can check the accuracy of integration by estimating the value of $\bar{H}$. This idea is applicable to the case of non-periodic time-dependent Hamiltonians.

\section{APPENDIX II \\ PROOF OF THEOREM 2}

The proof is based on the following two lemmas:

Lemma 3: $\operatorname{cl}\left(A_{\theta_{0}}\right) \times \mathbb{T}^{k}$ is uniformly homeomorphic to $\operatorname{cl}\left(\mathcal{O}\left(I_{\theta_{0}}\right)\right)$.

Proof: This proof is the direct application of the following corollary:

Corollay 1 (Corollary 3.46 in [17]): Let $X$ and $Y$ be complete metric space, and let $A$ and $B$ be dense subspaces of $X$ and $Y$, respectively. If $G: A \rightarrow B$ is a uniform homeomorphism of $A$ onto $B$, then there exists a unique uniform homeomorphism $\bar{G}: X \rightarrow Y$ of $X$ onto $Y$ that extends $G$ over $X$ (i.e., $\left.\bar{G}\right|_{A}=G$ ).

By definition, there exists a uniform homeomorphism of $A_{\theta_{0}} \times \mathbb{T}^{k}$ onto $\mathcal{O}\left(I_{\theta_{0}}\right)$. Then, by Corollary 1 , we see that the two closures $\operatorname{cl}\left(A_{\theta_{0}}\right) \times \mathbb{T}^{k}$ and $\operatorname{cl}\left(\mathcal{O}\left(I_{\theta_{0}}\right)\right)$ are uniformly homeomorphic.

\section{Lemma 4: $I=\operatorname{cl}\left(\mathcal{O}\left(I_{\theta_{0}}\right)\right)$.}

Proof: By construction we see $\mathcal{O}\left(I_{\theta_{0}}\right) \subset I$. Let $D$ be the difference set $I \backslash \mathcal{O}\left(I_{\theta_{0}}\right)$. Note that the interior of $D, \operatorname{int}(D)$, is empty. To prove this, we assume that $\operatorname{int}(D)$ is non-empty. Then, for some $x \in D$ there exists an open neighborhood $U$ such that $x \in U$ and $U \subset D$. From Fact 1, for $x=(m, \theta) \in D$ there exists a sequence of points, $\left\{\theta_{n}\right\}_{n=1}^{\infty}$, such that each $\theta_{n}$ is an element of $\left.\mathcal{O}\left(I_{\theta_{0}}\right)\right|_{\mathbb{T}^{k}}$ and it approaches to $\theta$. This is contradictory to the existence of open neighborhood of $U \subset D$. Hence we have $\operatorname{int}(D)=\emptyset$. Using the relation $(\operatorname{int}(D))^{\mathrm{c}}=\operatorname{cl}\left(D^{\mathrm{c}}\right)$, we obtain $I=\operatorname{cl}\left(\mathcal{O}\left(I_{\theta_{0}}\right)\right)$. 
We are now back to the proof of Theorem 2. If $A_{\theta_{0}}$ is bounded in $M$, then $\operatorname{cl}\left(A_{\theta_{0}}\right) \times \mathbb{T}^{k}$ is bounded in $X$. By Lemma $3, \operatorname{cl}\left(\mathcal{O}\left(I_{\theta_{0}}\right)\right)$ is bounded in $X$. By Lemma 4, we conclude that $I$ is bounded in $X$.

\section{REFERENCES}

[1] V. I. Arnold and A. Avez, Ergodic Problems of Classical Mechanics. Benjamin, 1968.

[2] J. P. Eckmann and D. Ruelle, "Ergodic theory of chaos and strange attractors," Reviews of Modern Physics, vol. 57, no. 3, pp. 617-656, July 1985

[3] S. Smale, "Differentialble dynamical systems," Bulltien of the American Mathematical Society, vol. 73, pp. 747-817, November 1967.

[4] D. D' Alessandro, M. Dahleh, and I. Mezić, "Control of mixing in fluid flow: A maximum entropy approach," IEEE Transactions on Automatic Control, vol. 44, no. 10, pp. 1852-1863, October 1999.

[5] I. Mezić, "Controllability, integrability and ergodicity," in Miltidiciplinary Research in Control, ser. LNCIS 289, L. Giarré and B. Bamieh, Eds. Springer-Verlag, 2003, pp. 213-229.

[6] P. G. Mehta, U. Vaidya, and A. Banaszuk, "Markov chains, entropy, and fundamental limitations in nonlinear stabilization," IEEE Transactions on Automatic Control, vol. 53, no. 3, pp. 784-791, August 2008.

[7] I. Mezić and S. Wiggins, "A method for visualization of invariant sets of dynamical systems based on the ergodic partition," CHAOS, vol. 9, no. 1, pp. 213-218, March 1999.

[8] K. Peterson, Ergodic Theory. Cambridge: Cambridge University Press, 1983.

[9] I. Mezić and A. Banaszuk, "Comparison of systems with complex behavior," Physica D, vol. 197, pp. 101-133, 2004.

[10] Z. Levnajić and I. Mezić, "Ergodic theory and visualization I: Visualization of ergodic partition and invariant sets," 2008, preprint: arXiv:0805/4221v1.

[11] M. Budišić and I. Mezić, "An approximate parametrization of the ergodic partition using time averaged observables," in Proceedings of the 48th IEEE Conference on Decision and Control, Shanghai, China, December 16-18 2008.

[12] S. Wiggins, Chaotic Transport in Dynamical Systems, ser. Interdisciplinary Applied Mathematics. New York: Springer-Verlag, 1992, vol. 2.

[13] Y. Susuki, I. Mezić, and T. Hikihara, "Global swing instability of multimachine power systems," in Proceedings of the 47th IEEE Conference on Decision and Control, Cancun, Mexico, December 911 2008, pp. 2487-2492.

[14] — , "Coherent swing instability of power grids," (in preparation).

[15] V. V. Nemytskii and V. V. Stepanov, Qualitative Theory of Differential Equations. Priceton: Princeton University Press, 1960.

[16] H. Yoshida, "Construction of higher order symplectic integrators," Physics Letters A, vol. 150, no. 5-7, pp. 262-268, November 121990.

[17] C. S. Kubrusly, Elements of Operator Theory. Boston: Birkhäuer, 2001. 